\title{
LAPAROSCOPIA NO ABDOME AGUDO INFLAMATÓRIO DE DIFÍCIL DIAGNÓSTICO
}

\author{
LAPAROSCOPY IN INFLAMATORY ACUTE ABDOMEN OF DIFFICULT \\ DIAGNOSIS
}

\author{
Antonio Carlos Valezi, TCBC - PR ${ }^{1}$ \\ Jorge Mali Junior ${ }^{2}$ \\ Rodrigo Gomes de Oliveira ${ }^{2}$ \\ Mario Liberatti ${ }^{3}$ \\ Antonio César Marson ${ }^{3}$ \\ Edivaldo Macedo de Brito ${ }^{4}$
}

\begin{abstract}
RESUMO: Objetivos: O objetivo deste estudo foi analisar a eficácia do método laparoscópico em casos de abdome agudo inflamatório de difícil avaliação, quanto à acurácia, sensibilidade, especificidade e valores preditivos positivo e negativo. Método: Foram examinados, prospectivamente, 29 doentes com suspeita clínica de abdome agudo inflamatório, que após exames clínico e complementares não esclarecedores, foram submetidos à laparoscopia diagnóstica e/ou terapêutica. Resultados: A precisão diagnóstica do exame foi de $96,5 \%$. Com relação à terapêutica, 58,6 \% dos doentes foram tratados por laparoscopia, 34,4\% clinicamente e $6,8 \%$ por laparotomia. A taxa de complicação foi de 10,3\%, com ausência de mortalidade nesta série. Os doentes submetidos ao tratamento laparoscópico, tiveram alta hospitalar em média 36 horas após o procedimento. Conclusões: A laparoscopia mostrou-se um método de elevada acurácia diagnóstica, que permitiu manejo terapêutico satisfatório, associado à baixa morbidez e à recuperação pósoperatória precoce.
\end{abstract}

Descritores: Abdome agudo; Laparoscopia; Técnicas de diagnóstico por cirurgia.

\section{INTRODUÇÃO}

O diagnóstico preciso do abdome agudo inflamatório torna-se, às vezes, tarefa difícil mesmo nas mãos de cirurgiões experientes, e com exames laboratoriais e de imagem adequados.

A laparoscopia tem se mostrado método diagnóstico e, às vezes,terapêutico, indicado nestes casos. ${ }^{1,2}$ Vários autores tem demonstrado uma elevada acurácia diagnóstica ( 86 a 100 \%) em pacientes submetidos à laparoscopia ${ }^{3,4}$.

A questão crucial na avaliação do abdome agudo inflamatório - operar ou não - tem na laparoscopia a melhor chance de resposta ${ }^{5}$.

O objetivo deste estudo foi avaliar a eficácia da laparoscopia no diagnóstico do abdome agudo inflamatório de difícil avaliação, bem como a sua capacidade de orientar a terapêutica.

1. Professor Adjunto Doutor do Departamento de Cirurgia da Universidade Estadual de Londrina

2. Médico Residente de Cirurgia Geral do Departamento de Cirurgia da Universidade Estadual de Londrina

3. Professor Assistente Mestre do Departamento de Cirurgia da Universidade Estadual de Londrina

4. Professor Adjunto Mestre do Departamento de Cirurgia da Universidade Estadual de Londrina

Recebido em 01/04/2002

Aceito para publicação em 03/06/2003

Trabalho realizado no Pronto Socorro Cirúrgico do Departamento de Cirurgia do Hospital Universitário da Universidade Estadual de Londrina. 


\section{MÉTODO}

Foram examinados 29 doentes, no período de 1997 à 2001, admitidos no pronto-socorro do Hospital Universitário da Universidade Estadual de Londrina, portadores de afecções abdominais agudas inflamatórias, que, após terem sido submetidos à avaliação inicial pela equipe de plantão e tendo permanecido dúvida quanto ao comprometimento abdominal, foram submetidos à laparoscopia.

Os exames laparoscópicos, realizados pelo mesmo examinador,foram feitos com laparoscópio Olympus dentro dos mesmos princípios utilizados quando o procedimento é feito eletivamente. Todos os doentes foram submetidos à anestesia geral.

Para a análise dos resultados foram considerados os valores de acurácia, sensibilidade, especificidade e valores preditivos positivo e negativo.

\section{RESULTADOS}

A média de idade foi de 32 anos, com variação entre 19 e 47 anos. Vinte pacientes eram do sexo feminino e nove do masculino. O tempo entre o início da dor abdominal e a realização da laparoscopia foi de 12 à 72 horas.

Dos 30 procedimentos, realizados em 29 doentes, o diagnóstico mais comum foi apendicite aguda, verificada em 12 doentes (41,3\%). Desses, 10 foram tratados por laparoscopia e dois foram convertidos, devido à abscesso grande e lesão de sigmoide por trocarte, que foi tratada por rafia e drenagem.

Em cinco pacientes o achado foi de anexite aguda.

Doença inflamatória pélvica foi diagnosticada em quatro ocasiões.

Abscesso tubo-ovariano foi encontrado em três pacientes e o tratamento foi drenagem.

Cisto de ovário roto em dois doentes e torcido em um, foram tratados por ooforectomia.

Necrose de epíplon em um paciente, foi tratada por ressecção.

A laparoscopia foi normal em dois pacientes. Em um destes, o mesmo procedimento foi realizado 24 horas após, pois o paciente permanecia com dor abdominal, e foi diagnosticado apendicite aguda.

Ocorreram três complicações operatórias laparoscópicas $(10,3 \%)$ na presente série: lesão de sigmóide por trocarte, abscesso subcutâneo e abscesso em fundo de saco posterior.

A conduta terapêutica foi clínica em 10 doentes $(34,4 \%)$, cirúrgica por laparotomia em dois pacientes $(6,8 \%)$ e por laparoscopia em $17(58,6 \%)$.

Nesta casuística a taxa de conversão foi de $10,5 \%$ (dois pacientes ). Os achados dos exames laparoscópicos encontram-se no quadro 1.

Os pacientes não operados foram observados por um período de 48 à 72 horas. Os submetidos à tratamento laparoscópico tiveram alta hospitalar em média 36 horas após o procedimento.

A acurácia foi de $96 \%$, a sensibilidade 96,5 $\%$, a especificidade $100 \%$, e valores preditivos positivo e negativo de $100 \%$ e $50 \%$, respectivamente.

Quadro 1 - Diagnósticos laparoscópicos em doentes portadores de afecções abdominais agudas inflamatórias.

\begin{tabular}{lr}
\hline Achados & No $^{\mathbf{0}}$ \\
\hline Apendicite aguda & 12 \\
Anexite aguda & 5 \\
Doença inflamatória pélvica & 4 \\
Abscesso tubo-ovariano & 3 \\
Cisto de ovário roto & 2 \\
Cisto de ovário torcido & 1 \\
Necrose de epíplon & 1 \\
Laparoscopia normal & 2 \\
\hline
\end{tabular}

\section{DISCUSSÃO}

A laparoscopia, utilizada há várias décadas , inicialmente era empregada para esclarecimento de afecções ginecólogicas. Atualmente, com o desenvolvimento dos equipamentos e sistemas ópticos, a laparoscopia teve ampla aceitação pelos cirurgiões do mundo todo, e passou a ser método terapêutico para diversas afecções abdominais. ${ }^{6-9}$

Empregada para tratamento de doenças eletivas, com o aperfeiçoamento técnico, os cirurgiões passaram a utiliza-lá no trauma ${ }^{10-13}$ e em doenças abdominais agudas. ${ }^{14-15}$

O abdome agudo inflamatório é a afecção abdominal mais comum no pronto-socorro cirúrgico, e a apendicite aguda a etiologia mais frequente.

Muitas vezes o quadro de abdome agudo inflamatório é de fácil diagnóstico,mas outras vezes, o 
mesmo torna-se muito difícil, levando a equipe que está atendendo o paciente, a deixá-lo em observação por tempo prolongado, a realizar exames onerosos e cirurgias desnecessárias. A utilização de um método diagnóstico que pudesse abreviar este tempo, seria muito vantajoso, e esta lacuna foi preenchida pela laparoscopia. ${ }^{6,16}$

O emprego da laparoscopia apresenta vantagens como, menos dor no pós-operatório, alta hospitalar precoce com retorno às atividade profissionais e propicia, além do diagnóstico, a possibilidade de tratamento.Foi possível realizar diagnóstico preciso em 28 pacientes $(96,5 \%)$, índice semelhante ao de outros estudos, que apresentaram elevada precisão diagnóstica ${ }^{3,4,6,17}$.

Com base nos dados referidos acima, naqueles casos de difícil diagnóstico, em pacientes sem cirurgias prévias, empregamos a laparoscopia sob anestesia geral.

Aponta-se como uma das desvantagens da laparoscopia, a dificuldade de realização de um inventário completo da cavidade abdominal e visualização direta de todos os órgãos,embora estu$\operatorname{dos}^{18}$ relatem que o delgado pode ser totalmente examinado por laparoscopia. Assim, em alguns pacientes portadores de apendicite aguda, o bloqueio de epiplon e de alças intestinais ou a situação anômala do apêndice, torna impossível a visualização do órgão. Tal fato fica ilustrado por um de nossos casos, em que o exame laparoscópico foi normal; como o paciente permanecia com dor abdominal, o examinador repetiu o exame e conseguiu localizar o apêndice cecal inflamado e de posição retrocecal, que foi prontamente ressecado.

Na nossa casuística a afecção predominante foi apendicite aguda e seu tratamento por laparoscopia foi possível em $80 \%$ dos casos. Índices de sucesso com a apendicectomia laparoscópica vão de 68 a $92 \%{ }^{3,4}$.

Em muitas ocasiões o diagnóstico de abdome agudo inflamatório nas mulheres torna-se um desafio, devido à grande variedade de urgências ginecológicas. Nesta série, $68,9 \%$ eram mulheres e 51,7\% dos diagnósticos foram de afecções ginecológicas. Além dos casos de processos inflamatórios agudos, como anexite ou doença inflamatória pélvica, que perfizeram nove casos nesta série, o exame permite o diagnóstico de outras afecções pélvicas de tratamento cirúrgico, como cisto de ovário torcido ou gravidez ectópica rota. Logo, além do diagnóstico, a laparoscopia orienta com precisão o tratamento mais adequado para cada caso de doença pélvica aguda, seja ele cirúrgico ou conservador. ${ }^{19}$

Cinco pacientes com anexite aguda, quatro com doença inflamatória pélvica e uma com exame normal, foram beneficiados pela laparoscopia, devido a não realização de cirurgia aberta para diagnóstico, evitando possivelmente $34,5 \%$ de laparotomias exploradoras. Este fato tem relevância, pois além de não terem sido expostos aos riscos inerentes de um ato operatório, tiveram alta hospitalar precoce. Além disso, a laparoscopia propiciou diagnóstico preciso e precoce e a instituição imediata da melhor conduta terapêutica para cada caso.

As complicações que apresentamos foram, lesão de sigmóide por trocarte, abscesso subcutâneo e abscesso em fundo de saco posterior, ressaltando aqui que a introdução dos trocartes é passível de produzir lesão de órgãos intracavitários, principalmente na primeira punção que é feita às cegas. A lesão foi identificada e tratada por laparotomia. Nossa taxa de complicação foi de $10,3 \%$ concordando com as taxas de complicações relatadas por outros autores $9,10,20$.

O insucesso da terapêutica laparoscópica pode ser avaliado pela taxa de conversão. Na literatura encontramos taxas de $2,7 \%$ à $39 \%^{3,16,17,21}$. Na presente série esta foi de 10,5\%.

Podemos concluir, portanto, que a laparoscopia apresenta baixo índice de complicações, sendo seu emprego seguro e de alta acurácia no diagnóstico e tratamento de afecções abdominais agudas, além de evitar atraso no diagnóstico e número significativo de laparotomias desnecessárias. Na maioria dos casos o tratamento definitivo pode ser realizado sem necessidade de conversão, com vantagens adicionais da cirurgia minimamente invasiva, como pequena resposta metabólica ao trauma e alta hospitalar precoce com retorno às atividades de trabalho.

Propomos, baseado neste estudo, a difusão desse método diagnóstico e/ou terapêutico, em instituições que prestam atendimento à pacientes na urgência. 


\begin{abstract}
Objective: The aim of this study is to analize the efficacy of laparoscopy in cases of acute inflamatory abdomen of difficult diagnosis, according to accuracy, sensitivity, specificity and negative and positive predictive values. Methods: The authors studied, prospectively, 29 patients with clinical suspicion of inflamatory acute abdomen, that, after inconclusive clinical and complemental investigations were submitted to diagnostic and / or therapeutic laparoscopy. Results: In 96,5\% of the patients the procedure confirmed the diagnosis; $58,6 \%$ of the patients were treated by laparoscopy, 6,8\% by laparotomy and $34,4 \%$ received clinical treatment. The complication rate was $10,3 \%$, with no mortality in this study. Patients treated by laparoscopy were discharged from hospital in 36 hours after the procedure. Conclusions: Laparoscopy proved to be a good diagnostic and therapeutic method in acute inflamatory diseases of the abdomen and is associated with lower hospitalization time and lower rate of complications.
\end{abstract}

Key Words: Acute, abdomen; Laparoscopy; Diagnostic techniques, surgical.

\section{REFERÊNCIAS}

1. Berci G, Dunkelman D, Michel SL, et al. - Emergency minilaparoscopy in abdominal trauma. Am J Surg, 1983, 146(2):261-265.

2. Cortesi N, Zambarda E, Manenti A, et al. - Laparoscopy in routine and emergency surger: experience with 1.720 cases. Am J Surg, 1979, 137(5): 647-649.

3. Navez B, d'Udekem Y, Cambier E, et al. - Laparoscopy for management of nontraumatic acute abdomen. World J Surg, 1995, 19(3): 382-387.

4. Paterson-Brown S - Emergency laparoscopy surgery. Br J Surg, 1993, 80(3): 279-283.

5. Pitombo MC, Guarino JL, Martins LA, et al. Laparoscopia no abdome agudo não traumático: estudo retrospectivo. Rev Col Bras Cir, 1999, 26(4): 233-236.

6. Cuesta MA, Eijsbouts QA, Gordijn RV, et al. - Diagnostic laparoscopy in patients with acute abdomen of uncertain etiology. Surg Endosc, 1998, 12(7): 915-917.

7. Memon MA, Fitzgibbons RJ - The role of minimal access surgery in the acute abdomen. Surg Clin North Am, 1997, 77(6): 1333-1353.

8. Rodrigues FCM, Solda SC, Silva RA, et al . - Lise de bridas pela cirurgia videolaparoscópica. Rev Col Bras Cir, 1994, 21(3): 132-135.

9. Valezi AC, Rahal F - Repercussões do pneumoperitônio sobre o sistema venoso dos membros inferiores - Estudo em porcas. Rev Col Bras Cir, 1999, 26(1): 45-49.

10. Solda SC, Rodrigues FC, Martins L, et al. - Lesão diafragmática isolada por ferimento penetrante tratada por videolaparoscopia. Rev Col Bras Cir, 1995, 21(4): 213-215.

11. Solda SC, Rodrigues FCM, Rasslan S - Videolaparoscopia diagnóstica nos ferimentos da transição toracoabdominal. Rev Col Bras Cir, 1996, 23(6): 307-311.

12. Solda SC, Rodrigues FCM, Silva RA, et al. Coleperitônio após colecistectomia convencional tra- tado por via laparoscópica. Rev Col Bras Cir, 1999, 26(1): 69-71.

13. Zantut LFC, Zantut PEC, Birolini D - Laparoscopia e autotransfusão em pacientes traumatizados. Estudo em 21 casos. Rev Col Bras Cir, 1991, 18(4): 139-142.

14. Connor TJ, Garcha IS, Ramshaw BJ, et al. - Diagnostic laparoscopy for suspected appendicitis. Am Surg, 1995, 61(2):187-189.

15. Poole GV, Thomas KR, Hauser CJ - Laparoscopy in trauma. Surg Clin North Am, 1996, 76(3): 547-556.

16. Moberg AC, Ahlberg G, Leijonmarck CE, et al. Diagnostic laparoscopy in 1043 patients with suspected acute appendicitis. Eur J Surg, 1998, 164(11): 833-840.

17. Cueto J, Diaz O, Garteiz D, et al. - The efficacy of laparoscopic surgery in the diagnosis and treatment of peritonitis. Experience with 107 cases in Mexico City. Surg Endosc, 1997, 11(4):366-370.

18. Kawahara N - Padronização dos procedimentos laparoscópicos no trauma abdominal penetrante: estudo de 75 casos. Dissertação (Doutorado em Cirurgia). São Paulo. Universidade de São Paulo, 1997, 89p.

19. Attwood SE, Hill AD, Murphy PG, et al. - A prospective randomized trial of laparoscopic versus open appendectomy. Surgery, 1992, 112(3): 497-501.

20. Chung RS, Diaz JJ, Chari V - Efficacy of routine laparoscopy for the acute abdomen. Surg Endosc, 1998, 12(3): 219-222.

21. Henry C, Smadja C, Vons C, et al. - Resultats du traitement coelioscopique des urgences abdominales. Ann Chir, 1998, 52(3): 223-228.

Endereço para correspondência:

Antonio Carlos Valezi

Rua Santos 777 apto 1302

86020-021 Londrina - PR

valezi@conectway.com.br 